\title{
Optimization of Wireless Ad-hoc Networks using an Adjacent Collaborative Directional MAC (ACDM) Protocol
}

\author{
Mohammad Samawat \\ Ullah \\ Faculty Member \\ American International \\ University-Bangladesh (AIUB)
}

\author{
Fernaz Narin Nur \\ Senior Lecturer \\ Daffodil International University
}

\author{
Nazmun Nessa Moon \\ Assistant Professor \\ Daffodil International University
}

\begin{abstract}
In this paper we designed a modern Medium access control (MAC) protocol for wireless ad-hoc network that focuses on neighbor node information availability and uses directional antenna. MAC protocol coordinates different users to share the wireless channel fairly and resourcefully in the wireless networks. However, using directional antennas in ad-hoc networks causes new challenges such as new hidden terminal, deafness problems, unnecessary blocking of nodes etc. The problems arise mostly due to lack of information of the neighbor node's activities. Thus, we proposed a new directional MAC protocol name Adjacent Collaborative Directional MAC Protocol (ACDM) for wireless ad-hoc networks. The objective is to improve the throughput and delay performance together with overhead reduction of the wireless network. In addition, the integrity of the ACDM has been verified using the distributed network simulator tool NS3. The simulation results have shown that the ACDM protocol outperforms the existing protocols of wireless networks using directional antennas by minimizing the depressing effect of hidden-terminal, deafness and head of line blocking problems that also avoids asymmetry-in-gain problem. The performance of ACDM protocol shows that it improves the throughput and reduces the overhead from the state-of-art works.
\end{abstract}

\section{Keywords}

ACDM, Directional Antenna, Medium Access Control, Adhoc Network, IEEE802.11, NAV, neighbor accommodating MAC, Collaborative MAC, Directional MAC.

\section{INTRODUCTION}

Wireless networks comprise a number of nodes spread out in an area. Nodes are connected by wireless channel without any infrastructure. IEEE 802.11 MAC is a carrier sense multiple accesses protocol with collision avoidance (CSMA/CA) mechanism which is used for several years to control the multiple access process of such networks. This protocol uses Omni-directional antennas for packet transmission. In these protocols, while two nodes are communicating with each other, the neighbor nodes have to stay quiet. Due to the presence of silent region, the network loses a large portion of channel capacity and this leads to low network throughput particularly in dense networks. An Omni-directional antenna has a $360^{\circ}$ coverage angle. It sends signals towards all horizontal directions $[1,2,20]$. However, since the energy is broadcasted in all directions and only a very small portion is actually received by the intended node, most of the transmitted energy is wasted [3]. Utilization of directional antennas is proposed in wireless networks in order to compensate this problem. Directional antennas allow several nodes to communicate with each other at the same time with minimum interference generation; since packets are sent in a particular desired direction, a neighbor node at the same direction may have to defer its transmission but the nodes in the other beams are still allowed to send and transmit. Thus, the spatial reuse of wireless channel increases dramatically. Again, directional antenna can spread the energy to a certain direction, so it decreases the energy consumed by the transmitter. In ad hoc networks with directional antennas the spatial reuse rate of wireless resources can be increased by configuring several nodes for interference-free communication simultaneously $[4,5]$. The local congestion and end-to-end delay can be decreased by such long-range links [5]. There are many applications in wireless networks that use directional antennas. For example, Vehicular networks and Millimeter-wave communications use directional antenna to overcome severe path loss [6].

In the wireless networks, as the channel is shared by all users, a medium access control (MAC) protocol is needed to coordinate different users to share the wireless channel fairly and resourcefully. The IEEE 802.11 DCF (Distributed Coordination Function) is a widely used MAC protocol for wireless communications [7, 8]. IEEE 802.11 was designed for Omni-directional antennas, but their carriers sense multiple accesses with collision avoidance (CSMA/CA) are the foundation of many directional MAC protocols. However, using directional antennas in ad-hoc networks cause new challenges in MAC protocols such as new hidden terminal, deafness problems and unnecessary blocking of nodes $[4,5$, 19]. These problems usually occur when a transmitter does not have any information about the neighbor node's activities $[1,4]$. These issues result mainly from the fact that the data transmission period of certain nodes may overlap the RTS/CTS (Request to Send / Clear to Send) exchange period of their adjacent nodes or of nodes that are located outside the antenna beam of the RTS/CTS exchange. Consequently, these nodes cannot overhear the RTS/CTS frame exchanges from their neighboring nodes as a result collision can happen among them.

Our design objective is to improve the network aggregate throughput by reducing interference among nodes. The proposed Adjacent Collaborative Directional MAC (ACDM) protocol helps to improve the throughput and delay performance and at the same time reduce overhead of the wireless network. In particular, the recommended ACDM protocol uses directional transmission and directional reception for data packets and also for control packets that minimizes the depressing effect of hidden-terminal, deafness and also avoids asymmetry-in-gain problem. 
The reminder of the paper is segmented into sections and section-2 briefly describes the literature review of related research works, section-3 describes the antenna model of the proposed system and the proposed protocol and the solutions to the problems in detail including the design and implementation details. Section-4 specifies the performance evaluation from the results of the solutions including the information on experimental settings together with benefits and disadvantages of the proposed solutions. Finally the conclusion and future potential researches are outlined in Section 5 .

\section{REVIEW OF RELATED LITERATURE}

While shared channel is used in directional sensor network, many channel allocation problem like hidden station problem, deafness problem can happen [1,2, and 3]. Because of these problems, the performance of network is directly affected and cause throughput degradations and unnecessarily wasted of the channels among nodes. Over the last few years several MAC protocols for ad-hoc networks have been proposed to solve these problems; still they demand more justifiable outcomes to solve these problems. In ad hoc networks random permission based MAC protocol design and analysis has involved extensive research works [9, 10, and 11].

A significant number of research efforts focus on adapting the IEEE 802.11 MAC to appropriately work with beam forming antennas. This scope of revisiting IEEE 802.11 was primarily limited to choose whether to perform the protocol operations with the antenna in direction or an Omni-direction mode. The DMAC [12] is one the earliest protocols that support directional antenna. This protocol is based on a modified 802.11 MAC protocol and uses per sector blocking mechanism to obstruct a sector as soon as it senses a RTS or CTS packet. The protocol requires knowledge of neighbors' location. A node can transmit its own RTS packet in an Omnidirectional fashion when none of its sectors is blocked. For using Omni-directional antenna, it causes unnecessary blocking. Another protocol [13] uses RTS/CTS packets in an Omni-directional fashion using all available sectors. After successful RTS/CTS handshake, the data and ACK are transmitted in the directions from which the CTS/RTS are received at the maximum power. This protocol is efficient in minimizing the hidden terminal problem but it creates a severer exposed terminal problem and also cannot handle the deafness problem. In MMAC [1] a multi hop RTS MAC protocol is proposed where all packets including RTS/CTS uses directional transmission (DRTS/DCTS ${ }^{1}$ ). Nodes may listen in an Omni-directional mode while they are idle. But in this protocol the deafness problem still remains as all neighboring nodes is unable to accept the DRTS and DCTS.

Moreover, a closely related research based on a cooperative MAC protocol (CoopMAC) sets an intermediary in the middle of transmitter and receiver to improve poor channel quality. The CoopMAC receives the traffic from receiver, stimulate the slow communication and send it towards the destination. Thus, it converts one-hop transmission into two-hop transmission resulting the decrease in transmission time. This is happens by one of few cooperative neighbors that transfers traffic in support of source station. While a neighbor with higher transfer rate helps in transmission, the overall network performance noticeably improves. Finally as the

\footnotetext{
${ }^{1}$ DRTS/DCTS = Directional Request to Send/Directional Clear to Send
}

communication process lasts less time, new transmission gets started early and therefore overall performance noticeably improves with the help of CoopMAC protocol [18]. Recently, many researchers have focused on proposing directional MAC protocols that resolve these beam forming related problems. The majority of the protocols handle the possibility of deafness problems by sending multiple directional control packets sequentially to inform neighbors about the ongoing transmission and some protocols addressed also the asymmetry-in-gain problem [15, 16, 17, and 18]. These approaches reduce deafness to some extent. However, the overhead of these protocols is high enough to limit the benefit of spatial reuse. Besides, the CoopMAC protocol [18] and its result outcome of cooperation strategies are substantial content to relate with our proposed ACDM protocol. To some extent, this is related to our proposal. Thus, it seemed more reliable to relate with ACDM neighbor accommodating method. Although CoopMAC focuses only on cooperative stations, we tried to relate and include our features in ACDM. Apart from these efforts, there are still many difficulties because of some significant problems that arise with the deployment of directional antennas. In particular, most of the suggested solutions do not avoid the requirement of Omnidirectional transmissions and receptions of control packets which results asymmetry-in-gain problem and also limits the frequency re-use capability which can decrease the network throughput.

\section{THE PROPOSED ACDM PROTOCOL}

We consider a wireless network composed of several nodes. All nodes in the network are equipped with adaptive array of antenna systems. Mainly, there are three types of directional antenna systems: switched beam antenna system, steered beam antenna system, and adaptive antenna system [8]. We assume that each node is equipped with a switched beam antenna system in the network as in Fig 1, which is comprised of $\mathrm{M}$ fixed beam patterns. In our model, we assume an antenna can work in two modes: Omni mode and directional mode. If nodes do not have anything to send out, the respective antennas work in Omni method to sense signals. It sends and receives data in directional mode. Nodes are prepared with GPS components to know their GPS geographic locations. Initially an idle node waits for signals in Omni mode. Once a signal is sensed in Omni mode, the antenna detects the beam to specified direction on which the signal power is strongest and goes into the Directional mode. In directional mode a node can direct its beam to a specific direction with gain $G^{d}>G^{0}[2,3,7]$.

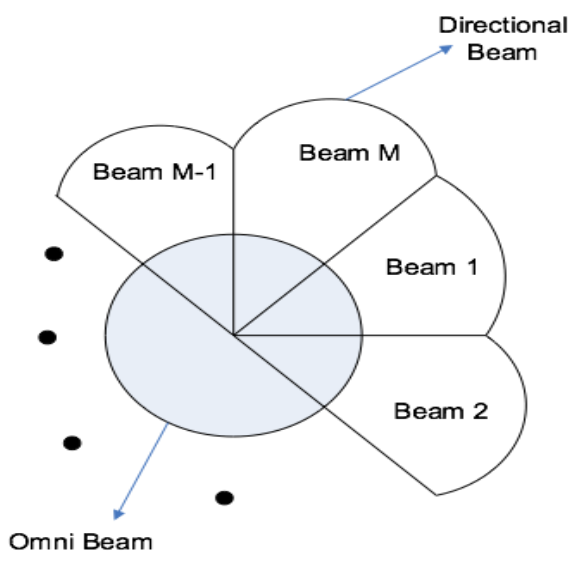

Figure 1: (a) Antenna Model, (b) Antenna Pattern 
We propose a directional MAC protocol to solve the hiddenterminal, exposed-terminal, deafness problems and reduce the overhead and exploit the benefits of directionality, e.g, spatial reuse, higher transmission range etc. The proposed ACDM protocol uses a single wireless data channel for transmission and reception. The channel carries the data packets and all other control packets on a specified direction. The protocol assumes that the directions of all reachable destinations or forwarders are predetermined during the node discovery period.

\subsection{Deafness Table}

In this ACDM protocol, every node keeps a Deafness table (DT), which contains the record for every node that it has heard and their corresponding periods for being busy. Initially the DT is empty and it is continuously updated upon overhearing any transmission.

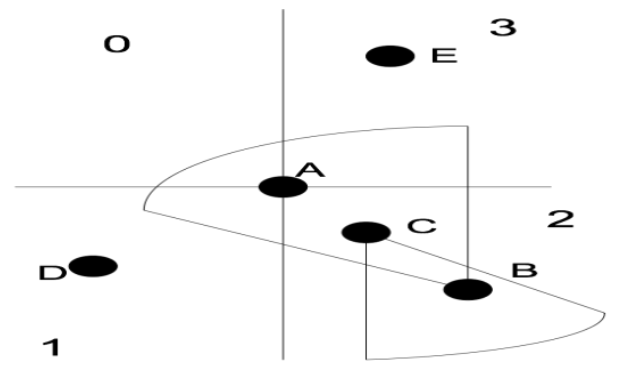

Figure 2: Example Scenario for Deafness Table

When a node receives a DRTS frame and the receiver address matches its address, it beamforms toward the transmitter and replies the DRTS with a DCTS frame. If the control frames are not for it or it receives a DWTS frame, it will update the sender's information in the DT and set the corresponding Directional Network Allocation Vectors (DNAVs).

Table 1. Deafness Table for node A

\begin{tabular}{|c|c|c|c|}
\hline Neighbors & Beam No. & Available & Duration \\
\hline- & 0 & Yes & \\
\hline D & 1 & Yes & \\
\hline B & 2 & No & $10 \mathrm{~ms}$ \\
\hline C & 2 & No & $10 \mathrm{~ms}$ \\
\hline E & 3 & Yes & \\
\hline
\end{tabular}

The table 1 shows the structure of the Deafness table. Here from Figure 2, A's neighbor table is shown where B and C are communicating with each other in beam number 2 so they can't initiate any communication with other node. So, Deafness table shows that B and $\mathrm{C}$ nodes are busy for $10 \mathrm{~ms}$ and if node $\mathrm{A}$ wants to communicate with $\mathrm{B}$ or $\mathrm{C}$, it has to try after $10 \mathrm{~ms}$. From the DWTS ${ }^{2}$ frame, each node updated their Deafness table. With this DT the deafness problem could be avoided.

\footnotetext{
${ }^{2}$ DWTS $=$ Directional Wait To Sent
}

\subsection{Directional Network Allocation Vectors (DNAV) mechanism}

Directional Network Allocation Vector (DNAV) is addressed as a directional version of NAV of IEEE 802.11. DNAV does not consider the directions and sets the corresponding durations, toward which the node is not permitted to begin transmission to avoid collisions with data. An example scenario is given in Fig 3.

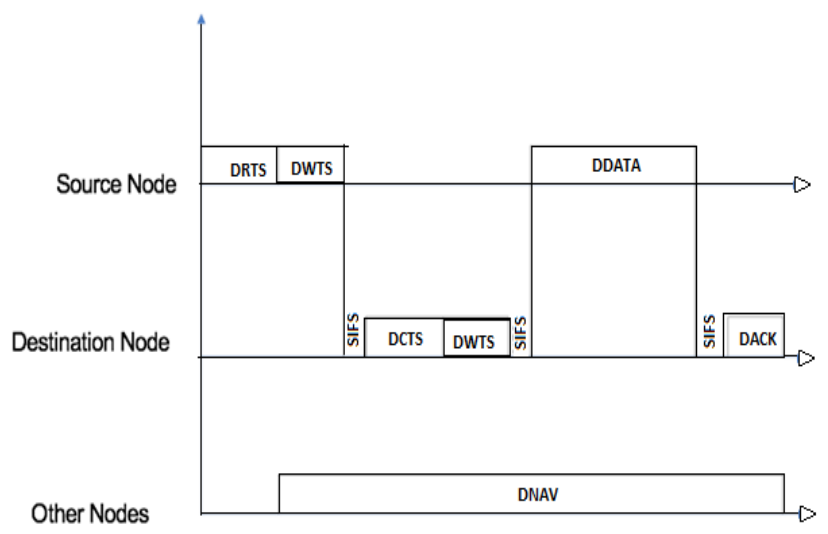

Figure 3: DNAV Timing diagram

Fig. 3 shows an example of data communication among nodes. When a sender sends a DRTS message to a receiver node, it waits a predetermined DWTS time for receiving the DCTS. In the DWTS period, the sender sends the DRTS message to the other directions so that all surrounding nodes can hear the DRTS. After receiving the DCTS and waiting a DWTS time period the data transmission among sender and receiver take place. The other surrounding nodes who hears the DRTS/DCTS, update their deafness table, and after the DNAV period the other nodes try to access the medium.

\subsection{Transmitting and Receiving Mechanism}

While wireless node's link layer obtains data packets from higher level, it senses the action on the data channel at the specified direction. In this protocol, when any node has a packet to be sent towards other node(s), at first it performs physical carrier sensing. If the channel remains idle during back off periods, the transmitter node determines the beam in which potential transmitter exists. It then checks the own deafness table (DT) and also DNAV, for each beam to determine where potential transmitters are located and DNAV is not set in its beam. The sender node then sends a DRTS (Directional Ready To Send) towards the receiver using a particular beam. Then the sender node sends the DWTS (Directional Wait To Send) to all the other beams so that the surrounding neighboring nodes can update their Deafness Table and defer their transmission to the same channel. We integrated the DNAV mechanism with the Deafness Table. When a receiver node receives a DRTS frame and the receiver address matches its address, it switches to directional mode and beam forms toward the transmitter and replies the DRTS with a DCTS frame. If the control frames do not belong to the node or the node receives a DWTS packet, it will update the sender's information in the Deafness Table and set the corresponding DNAVs.

In the protocol, each transmitter contends the channel by sending a DRTS frame to the receiver. The frame format of DWTS is same as the DRTS. After the receiver receives the DRTS frame successfully, the receiver replies with a DCTS 
frame. In addition, the receiver sends DWTS frame towards the nodes changing it's beam to other directions. Again, the frame format of DWTS is same as the DCTS. In DWTS the sender and receiver also mention the time period for contending the channel so that surrounding nodes that receive the DWTS can update their deafness table. After the successful hand-shaking, the data transmission will proceed immediately. All the other nodes receiving DWTS will set their DNAV values and defer the channel access until the end of the transmission.

If required time frame for sending DRTS is $t$ and there are $n$ sectors then the required time for sending DRTS to all directions is $\mathrm{n} \times \mathrm{t}$. Thus, as soon as the receiver gets the DRTS, it will send the DCTS after $\mathrm{n} x \mathrm{t}$ time period, so that at that time the sender is beam forwarded to receiver and gets the DCTS. After that the receiver will also send the DWTS to all other directions. When the adjacent nodes receive the DWTS, these nodes set their DNAV according to DWTS and defer their own transmissions addressed to that node to avoid collision until the entire data transmission is completed. After the successful handshaking, the source node sends the directional data frame and the receiver node sends the directional ACK frame. Both nodes switch back to the idle mode after the DATA/ACK frame exchange. In the idle mode the nodes will be in Omni-directional mode and listens the channel. Again, if the neighbor node fails to communicate with the sender of DWTS and the backoff procedure is invoked before receiving DWTS, it discards the frozen backoff counter and reselects a new backoff counter from [0, $\left.\mathrm{CW}_{\min }\right]$ for the next attempt. The ACDM mechanism is as follows:

1. Begin

2. Check if the channel is idle?

3. IF true THEN wait DIFS ${ }^{3}$ time and Jump to the step-5.

4. IF false THEN set back-off timer and Jump to the step-2

5. Check if the channel is still idle?

6. IF true THEN wait DIFS time and Jump to the step8.

7. IF false THEN set back-off timer and Jump to the step-2

8. Send DRTS to Receiver

9. Send DRTS to all other neighbors.

10. Check whether the DCTS received before time-out?

11. IF true THEN wait SIFS ${ }^{4}$ time and Jump to the step13 .

12. IF false THEN wait back-off time and Jump to the step-2.

13. Send the data frame directionally

14. Check whether the DACK ${ }^{5}$ received before timeout?

${ }^{3}$ DIFS $=$ DCF interframe space

${ }^{4}$ SIFS $=$ Short interframe space

${ }^{5}$ DACK $=$ Directional acknowledgement
15. IF true THEN return Success and Jump to the step-20

16. IF false THEN Increment back-off timer.

17. Check if the back-off time limit exceeded?

18. IF true THEN abort the process and jump to step20.

19. IF false THEN wait back-off and Jump to step-5

20. Exit.

\section{ADDRESSING THE CHALLENGES} USING ACDM PROTOCOL

Firstly we outlined the general idea and the problems related to the Directional transmission in Directional Ad-hoc Network.

\subsection{Hidden and exposed station problem}

In directional ad-hoc networks, the hidden terminals are situated near to the source node. Tentatively, all nodes those are located surrounded by the destination node's coverage region and are far from the source node's coverage area are hidden terminals. In other word, hidden terminal problems arise when the transmitter and receiver have not listened to the DRTS or DCTS.

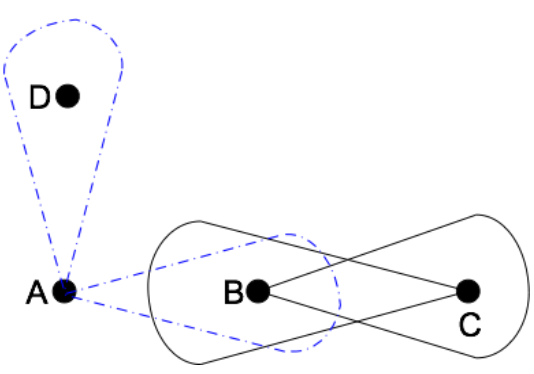

Figure 4: Hidden Terminal Problem

For illustration, in Fig 4 while node B is sending DRTS to node $\mathrm{C}$, node $\mathrm{A}$ is busy in communication with D. Node C's DCTS cannot reach to node A, as it is beam formed in the direction of $\mathrm{D}$. When the communication between nodes $\mathrm{B}$ and $\mathrm{C}$ is in progress, if has completed its transferring to $\mathrm{D}$ and has a packet to send to node $\mathrm{B}$. during that time $\mathrm{A}$ initiates messaging with B by sending DRTS to B, and it will lead to a collision with B's transmission. Hidden terminals can severely degrade the network performance. Regrettably, the standard RTS/CTS mechanism fall short to completely resolve the problem, as nodes near to the source node may begin transmissions during the time the source node transmits the RTS.

\subsection{Deafness Problem}

The Deafness problem occurs mainly in ad hoc networks where the nodes use directional antenna. If a source node fails to communicate with receiver which is pointing at a different location, deafness takes place.

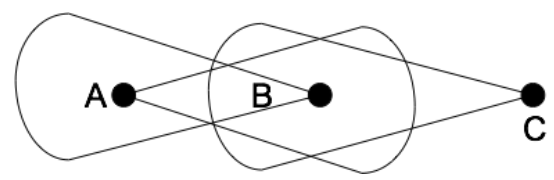

Figure 5: Deafness Problem

In Figure 5, node A and B is communicating with each other. Node $\mathrm{C}$ is trying to initialize communication with node B 
where node $\mathrm{B}$ is beaformed to node $\mathrm{A}$. For that, node $\mathrm{C}$ will not get DCTS in time and twice its backoff time for retransmission, since it shows that a conflict has occurred. When node $\mathrm{C}$ reaches its retry limit, it concludes that node $\mathrm{B}$ is unreachable.

\subsection{Asymmetry-in-Gain Problem}

When a directional transmission for data packets and an omnidirectional transmission for control packets such as RTS/CTS are used, different communication ranges direct to the asymmetry-in-Gain problem because the transmission range of a directed signal and the transmission range of an omnidirectional signal are not identical.

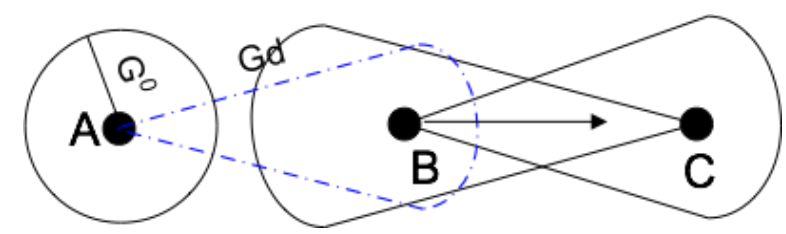

Figure 6: Asymmetry In Gain Problem

In Figure 6, when the nodes are in idle mode they listen omnidirectionally with a gain of $\mathrm{G}^{0}$. For example, B initiates transmission to node $\mathrm{C}$ by sending DRTS. Then node $\mathrm{C}$ sends DCTS to node $\mathrm{B}$. At that moment node $\mathrm{A}$ is in omnidirectional mode as it is in idle state. Node A is far enough away from node $\mathrm{C}$, so A cannot hear the DCTS from C because the omni-directional gain $G^{0}$ is smaller than the directional gain $\mathrm{Gd}$. When nodes $\mathrm{B}$ and $\mathrm{C}$ begin data transmission by pointing their transmission and reception beams to each other with a gain $G^{\mathrm{d}}$, at the same time A also wants to communicate with B using DRTS which interfere the ongoing data transmission.

\section{PERFORMANCE EVALUATION}

In this segment, we assess the performance of our proposed mechanism in a discrete event network simulator ns-3 in terms of the defined metrics. Each of our simulations runs for 1000 s and we have taken the average computation over 10 simulation runs for each graph. We deploy the sensors uniformly in a region of $1000 \times 1000 \mathrm{~m}^{2}$. The network configuration factors are shown in Table 2.

Table 2: Network Configuration Parameters

\begin{tabular}{|l|c|}
\hline \multicolumn{1}{|c|}{ Parameters } & Value \\
\hline Simulation Area & $1000 \mathrm{~m} \times 1000 \mathrm{~m}$ \\
\hline \& \$Deployment Type & Uniform random \\
\hline Number of Sensor Nodes & 1000 \\
\hline $\begin{array}{l}\text { Number of Communication and } \\
\text { Sensing Sectors }\end{array}$ & $2 \sim 6$ \\
\hline Transmission Range & $100 \mathrm{~m}$ \\
\hline Sensing Range & $50 \mathrm{~m}$ \\
\hline Packet Size & 64 Bytes \\
\hline Routing Protocol & AODV \\
\hline Simulation Time & 1000 Seconds \\
\hline
\end{tabular}

\subsection{Simulation Results}

We examined the behavior of the proposed protocol and compare the results with DMAC [12] protocol.

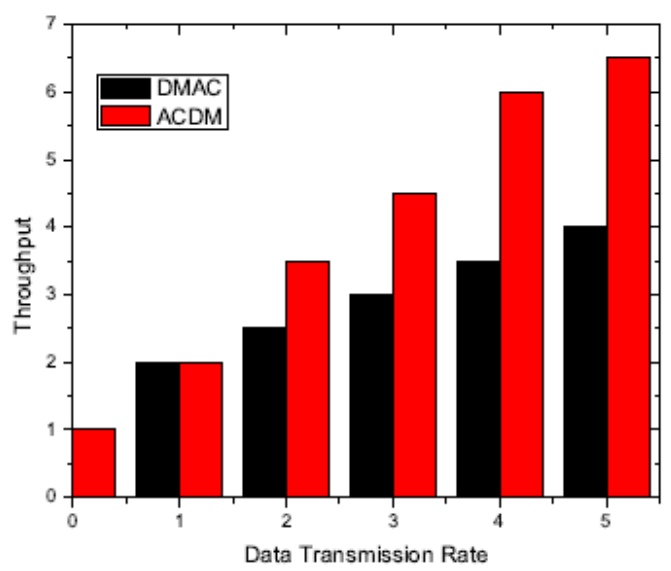

Figure 7a: Performances in the static random topology scenario: Total throughput.

The throughput is measured as the number of received data bytes second during the simulation period. The higher the value, better the MAC protocol performance is. Figure 7(a) shows the comparison of the total throughput. The result shows that the ACDM protocol gives better throughput than DMAC as the ACDM protocol uses directional transmission and directional transmission for all the control packets and data packets. Finally, we can say that in the ACDM, the throughput is increased about $40 \%$ than that of DMAC.

The end-to-end delay is defined as the duration from when the data packet is generated by the application layer of the source node to when the data packet is received by the application layer of the destination node, and discarded packets are not included in the delay calculation. Figure 7(b) gives the end-toend delay comparison. Because the number of hops from source node to destination node is efficiently reduced by the ACDM, its end-to-end delay is always lower than the DMAC's. From the simulation result we can say that our proposed ACDM protocol, after some time the end-to-end delay becomes constant as all the packets reach to the destination node with constant delay, but in the DMAC protocol the asymmetry in gain problem can cause more and more end-to-end delay.

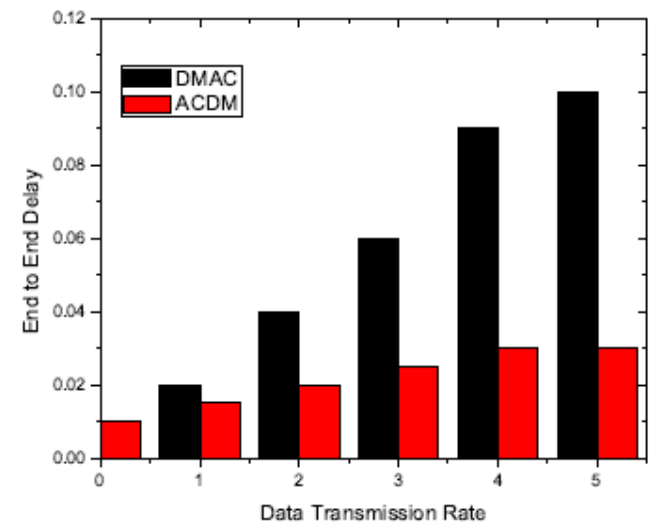

Figure 7b: Performances in the static random topology scenario: End-to-end delay. 


\section{CONCLUSION AND FUTURE WORK}

This research paper has proposed a new directional MAC protocol named as ACDM for wireless adhoc networks. It also critically analyzed the related literatures. Our experiments show that, the new protocol resolves the hidden-terminal, deafness problems, and also avoids unnecessary blocking. The surrounded nodes which are not in the transmission or reception beams can send/receive their data packets using different antenna beams. Moreover, the integrity of the ACDM has been verified using the distributed network simulator tool NS-3. Our performance analysis results based on UAT data shows that, and simulation results have shown that the ACDM protocol outperforms the existing protocols. The proposed protocol is based on static network model. In the future work, we will consider mobility model of the proposed protocol. The ACDM protocol does not cover the QoS metrics of MAC protocol. As it was beyond our project scope and due to some resource limitation we could not explore these issues and this is considered as one of our potential extension in future work.

The key motivation for ACDM protocol was to improve the wireless network performance using directional antennas. The proposed protocol helps to improve the throughput and delay performance of wireless ad-hoc network and at the same time reduces overhead. Based on the experimental results we believe that ACDM protocol will greatly improve as it reduces the unnecessary blocking and protocol overhead.

\section{ACKNOWLEDGMENTS}

Our special gratitude goes to all researchers who have contributed in wireless ad-hoc technology. In particular, thanks to the experts in our reference list. Their research works were really useful to conduct our analysis and accomplish the goal.

\section{REFERENCES}

[1] Romit Roy Choudhury, Xue Yang, Ram Ra-manathan, and Nitin H. Vaidya. Using directional antennas for medium access control in ad hoc net-works. In Proceedings of the 8th Annual International Conference on Mobile Computing and Net-working, MobiCom '02, pages 59-70, 2002.

[2] A. Spyrop oulos and C.S. Raghavendra. Energy efficient communications in ad ho c networks using directional antennas. In INFOCOM 2002. Twenty-First Annual Joint Conferences of the IEEE Computer and Communications Societies. Proceedings. IEEE, volume 1, pages 220-228 vol.1, 2002.

[3] Su Yi, Yong Pei, and Shivkumar Kalyanaraman. On the capacity improvement of ad hoc wireless networks using directional antennas. In Proceedings of the 4th ACM International Symposium on Mo-bile Ad Hoc Networking \&Amp; Computing, Mobi-Ho c '03, pages 108-116, New York, NY, USA, 2003. ACM.

[4] Young-Bae Ko, V. Shankarkumar, and N.F. Vaidya. Medium access control proto cols using directional antennas in ad ho c networks. In INFO-COM 2000. Nineteenth Annual Joint Conference of the IEEE Computer and Communications Societies. Proceedings. IEEE, volume 1, pages 13-21 vol.1, 2000.

[5] Ram Ramanathan. On the performance of Adhoc networks with beam forming antennas. In Proceedings of the 2Nd ACM International Symposium on Mobile AdHoc Networking \&Amp; Computing, Mo-biHoc '01, pages 95-105. ACM, 2001.

[6] Lichun Bao and J. J. Garcia-Luna-Aceves Receiveroriented multiple accesses in adhoc networks with directional antennas. Wirel. Netw, 11(1-2):67-79, January 2005.

[7] E. Shihab, Lin Cai, and Jianping Pan. A distributed directional-to-directional Mac protocol for asynchronous ad ho $\mathrm{c}$ networks. In Global Telecommunications Conference, 2008. IEEE GLOBECOM 2008. IEEE, pages 1-5, Nov 2008.

[8] Lichun Bao and J. J. Garcia-Luna-Aceves Receiveroriented multiple access in adhoc networks with directional antennas. Wirel. Netw.,11(1-2):67-79, January 2005.

[9] B. Kannhavong, H. Nakayama, Y. Nemoto,N. Kato, and A. Jamalip. A survey of routing attacks in mobile ad hoc networks. Wireless Communications, IEEE, 14(5):8591, October 2007.

[10] G. Bianchi. Performance analysis of the ieee 802.11distributed co ordination function. Selected Areas in Communications, IEEE Journal on, 18(3):535-547, March 2000.

[11] Thanasis Korakis, Gentian Jakllari, and Leandros Tassiulas. A Mac protocol for full exploitation of directional antennas in ad-ho $\mathrm{c}$ wireless networks. In Proceedings of the 4th ACM International Symposium on Mobile Ad Hoc Networking \&Amp; Computing, MobiHoc '03, pages 98-107, New York, NY, USA, 2003. ACM.

[12] Young-Bae Ko, V. Shankarkumar, and N.F.Vaidya. Medium access control proto cols using directional antennas in ad ho c networks. In INFO-COM 2000. Nineteenth Annual Joint Conference of the IEEE Computer and Communications Societies. Proceedings, IEEE, volume 1, pages 13-21vol.1, 2000.

[13] A. Nasipuri, S. Ye, J. You, and R.E. Hiromoto A Mac protocol for mobile adhoc networks using directional antennas. In Wireless Communications and Networking Conference, 2000. WCNC. 2000 IEEE, volume 3, pages 1214-1219 vol.3, 2000.

[14] R.R. Choudhury and N.F. Vaidya. Deafness: a Mac problem in adhoc networks when using directional antennas. In Network Protocols, 2004. ICNP 2004. Proceedings of the 12th IEEE International Conference on, pages 283-292, Oct 2004.

[15] M. Takata, M. Bandai, and T. Watanab e. A Mac protocol with directional antennas for deafness avoidance in ad ho c networks. In Global Telecommunications Conference, 2007. GLOBECOM '07. IEEE, pages 620625, Nov 2007.

[16] T. Korakis, G. Jakllari, and L. Tassiulas. Cdr-Mac: A protocol for full exploitation of directional antennas in ad ho $\mathrm{c}$ wireless networks. Mobile Computing, IEEE Transactions on, 7(2):145-155, Feb 2008.

[17] M. Takata, M. Bandai, and T. Watanab e. A receiverinitiated directional Mac protocol for handling deafness in adhoc networks. In Communications, 2006. ICC '06. 
IEEE International Conference on, volume 9, pages 4089-4095, June 2006.

[18] K. Thanasis, T. Zhifeng, S. Yevgeniy and P. Shivendra. A Cooperative MAC protocol for Ad Hoc Wireless Networks. In the Fifth Annual IEEE International Conference on Pervasive Computing and Communications Workshops, 0-7695-2788-4/07, 2007.
[19] Sudipta Mojumder, Syed Emdadul Haque, and Fernaz Narin Nur. "Directional MAC protocols in Ad-hoc Networks." International Journal of Computer Applications 100 (2014).

[20] Fernaz Narin Nur, Nazmun Nessa Moon and Narayan Ranjan Chakraborty. "A Survey on Routing Protocols in Wireless Multimedia Sensor Networks.” International Journal of Computer Applications 73 (2013). 